\title{
Global Account Management for Sales Organization in Multinational Companies*
}

\author{
Tino Canegrati ${ }^{* *}$
}

\begin{abstract}
A Global Company is not just a Multinational Company, but on top it has developed an organizational structure, an overall governance and a set of operational decision making processes which allow running a significant percentage of business processes as a unique body across borders.

Successful Global Companies have a clear setup and governance of local versus centralized decision making processes, as well as budget ownership

Corporations focused on global opportunities need to re-think their customer approach, namely in terms of set up of their sales force, and more specifically planning, a transformation from national based to international managed sales organization for those specific customers.
\end{abstract}

Keywords: Market-Driven Management; Global Companies; Multinational Companies; Vision; Global Account Management; Sales Organization; Corporate Culture; Corporate Responsibility

\section{Business Globalization}

A number of businesses have become global in the past few years, and many others are moving to the same direction. Everybody is aware that the main drivers for this evolution are related to lower barriers to commerce, facilitated communication and ideas exchange; everybody can see an overall trend towards more homogenous lifestyle among consumers from different countries and cultures, and a more standardized set of business practices in entrepreneurship world.

Standardization of products and processes are the first outcome of globalization; but they act themselves as a catalyst, driving economy of scales in design, production and distribution. And economies of scale act as boosters for further globalization, providing advantages to companies which are able to leverage their

\footnotetext{
* Invited Article

*** Director of University Relations HP - Technology Solutions Group EMEA (info@ hp.com)
} 
broad geographical presence to create even more economy of scale, or to complement their size and geographical coverage through merge and acquisition. The combination of these actions drives even more companies to consider the opportunity to expand their global strengths, by implementing changes in their organizations, both in R\&D and Production, but also in Distribution and Sales.

It happens more and more frequently that a successful national sales organization get impacted by the acquisition of one of their major accounts from an international company (and consequential changes in decision making processes); or, on the opposite extreme, that the same sales organization cannot access additional business opportunities generated from geographical expansion from one of their customers, because of the lack of coordination between different regions' sales forces. This paper wants to reflect on the challenges and the opportunities that such changes offer to sales organizations, and highlight potential ways of addressing them.

\section{Multinational Companies and Global Companies}

Without going in too many theoretical details on defining a Multinational or a Global Company, we want to address the main differences, specifically in light of their decision processes.

We know that Multinational Companies manage significant component of their turnover outside of their home countries, and across a significant number of subregions. We also have as a common perception that strong branding across countries and continents reinforces the concept of Multinational Companies. On the other side it is worth to reflect on the fact that not all Multinational Companies act as Global Companies. A Global Company is not just a Multinational Company, but on top it has developed an organizational structure, an overall governance and a set of operational decision making processes which allow running a significant percentage of business processes as a unique body across borders. A well set and run Global Company designs, runs and manages operational business processes while creating methodologies and efficiency standards which maximize the economy of scale of their business size; and it balances that standardization by keeping a dedicated local behavior wherever there is value added. Without naming them, each of us have in mind brands that they consider close to their daily life, while being aware that their operations run consistently and homogenously across dozens (or hundreds) of different countries. Successful Global Companies have a clear setup and governance of local versus centralized decision making processes, as well as budget ownership; Sales Organizations need to be very aware that transactional sales (and over time also part of solution sales) have standards defined at centralized level, and more and more are driven from centralized organizations. Concept of internal shared services in Procurement, IT, Real Estate, Finance, HR and other functions are implemented broadly in Global Companies, as they drive centralized, or at least strongly coordinated, budget ownership and decision making. Let's see together which reflections we suggest to a Sales Organization dealing with Global Companies. 


\section{Corporate Responsibility and Corporate Culture}

As seen in previous chapter, Global Companies have most of their procurement decision processes which are "centrally" driven through practices and standards designed and implemented across countries; moreover, most of decision making points are owned, or strongly influenced, by organizations which are not any more managed at local level. Does that mean that Sales Organizations should approach all Global Companies as Global Account? (i.e. creating for each of those Global Accounts a transnational sales organization)

Our perspective is that this is not a straight forward consequence. An International Sales Force which is in the process of evaluating if and how they have to change their Client Account structure from a national to a global one, should take in consideration a few major criteria:

- overall relevance of each account (both in terms of size of existing and potential business);

- "share of wallet" within each account (which represents both the relevance of their company (as a supplier) to the account, as well as the addressable additional opportunity;

- offer portfolio (i.e. transactional sales vs. solution/project sales);

- congruence between their sales organization and account decision making structure;

- competitors behaviour.

After having performed a thorough screening of customers' portfolio, and having identified companies which present behaviors peculiar to Global Companies, it is time to filter across those highlighted criteria: final goal being to identify those Global Accounts which look the most attractive for setting up a dedicated Sales Organization. Specifically it is very important to identify expected benefits from the setup of a dedicated Global Account Organization as well as to size goals which need to be achieved. Those benefits should be compared and weighted along with investments required to manage organizational changes and associated tuning in organizational, operational and financial reporting structure.

\section{Implementation of a Global Account Management Organization}

Once the preliminary analysis about the opportunity to set up a sales organization focused on Global Account has been performed, and the business case in terms of overall financials, ROI and risks mitigation has been found solid and sustainable, it is time to plan real deployment of the new structure, and start the transformation process.

While preparing the implementation of such a major change, a Sales Organization should not forget 3 major dimensions:

- the need of getting understanding and acceptance of members of an organization spread across dozens (if not hundreds) of countries; 
- the importance of proactively communicate to involved customers, as well to coordinate the deployment effort with them;

- the absolute must of planning and managing carefully such a transition, by driving operational changes firmly but smoothly and by rigorously making all major KPI (Key Performance Indicators) easily measurable and easy to track.

Planning an organizational change of such magnitude required to address at least 5 major steps:

- creating a Vision, i.e. an end point to be achieved;

- define and implement a Transformation Strategy, which allows to move from current setup to a defined end point;

- address MOC (Management of Change) as the key success factor for involving and mobilizing the whole population involved in the new setup;

- deploy the plan as defined, and carefully monitor progression;

- learn-as-you-go from obstacles encountered along the way while leverage from success.

Figure 1: Implementation Path for a Global Account Management Model

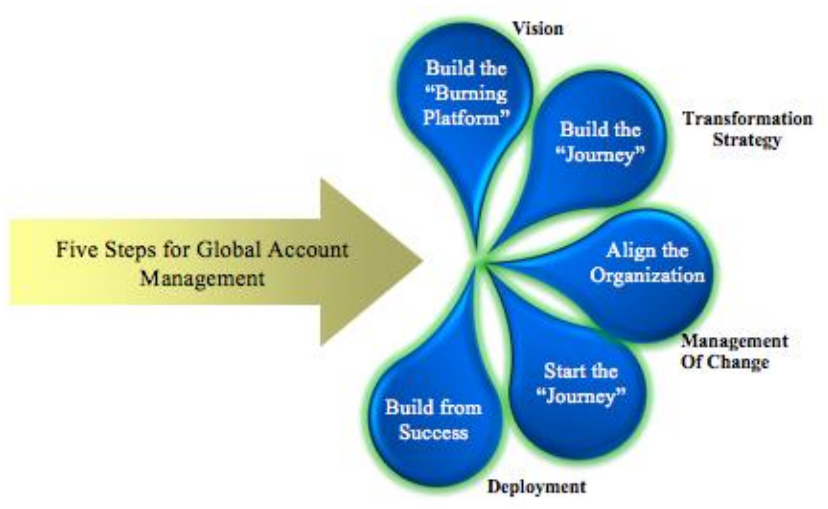

\subsection{Vision}

Definition of a Vision for the new organization it's the first, mandatory step. Vision should include associated expected benefits, tailored around returns for the company, advantages for customers, and reward for individual members of the sales teams.

End state has to be clearly defined and detailed in terms of organizational setup, ownership and responsibility of goals and objectives, operational frame and need to be complemented by identified and sized expected returns.

A thoroughly articulated and clearly communicated "burning platform" (the main compelling reasons behind a request for a massive organizational change) has to be prepared. Burning platform should include the compelling reasons why change is required now, and cannot be postponed.

A clear picture of the current and expected situation will help to mobilize the whole organization and to create the right sense of urgency. Identification of KPIs (Key Performance Indicators) such as revenues, profit, customer satisfaction and 
"share of wallet" will make the vision real and measurable. KPI historical trends, as well as related future objectives should be clearly defined and shared with all stakeholders and affected entities. Communication should also include relative current and expected position versus major competitors.

Country sales organizations and country account team members need to be involved in dedicated communication sessions, where a factual picture of current issues and constrains, and a desired and objective "end state", can be shared and discussed. Open, two-way discussion is mandatory for a successful acceptance and for gaining strong commitment from local sales forces (more comments on this point will be included in the session 4.3 "MOC - Management of Change").

\subsection{Transformation Strategy}

Vision should be supported by a well-thought, articulated and consistent Transformation Strategy, developed with an end-to-end perspective (so including, for example, actions associated to a refocus of marketing organizations: new setup and tailored operations need be in line with the new global dimension. As another example, it should include a detailed transition plan from current management reporting system to a new one, tuned to the new global customer centric perspective).

Transformation Strategy need to be at detailed level, to cover all components of the organization, while flexible enough to incorporate changes generated by "learning-as-you-go" along the way. Transformation should actually be considered as a journey: and as such, should be based on accurate planning, intermediate milestone precisely monitored and checked, clear and sharp final objectives. On the other side, management team driving transformation path needs to pay attention to all changes and unexpected obstacles or opportunities which will show up along the way; quick tuning and adjustments are key for success.

As mentioned such a long and complex journey requires that transformation address all operational components of organization, in order to make sure that business continuity is ensured and maintained across all steps. Particular attention needs to be devoted to data integrity, as well as to quality and frequency of reporting of those operational data which are mandatory to run business on a dayby-day basis (as an example revenues and profitability by project and by account; or a consolidated view of goods demand/supply picture across countries and subregions).

Methodologies for designing organizational changes (and specifically aimed to check congruency among formal and informal organizations, work to be done and people performing that work) have proven to be very useful, and can prevent major discrepancies (see for example David Nadler, Champions of Change)

Focus and attention to details, specifically to all areas which are critical to drive customer satisfaction and revenue generation, are mandatory for designing a successful Transformation Strategy.

Once this second component has been successfully addressed, there is another fundamental step which needs to be considered and carefully prepared: MOC. 


\subsection{MOC (Management of Change)}

Setting-up a Global Account Management Organization requires a systematic preparation that we have tried to summarize in the previous couple of paragraphs. But by itself a clear "end state" and a thorough preparation are not sufficient: it needs to be coupled with a comprehensive approach to inform and communicate to all involved stakeholders, from CEO to sales reps in the most remote area of the world. Success of a transformation of this magnitude requires that each member of involved organizations has a full understanding about:

- background which have urged the change (what we have called "burning platform");

- objectives and benefits which are targeted (as components of the what we have called Vision);

- expected contribution/involvement from him/herself as individual;

- how (overall/personal) success will be tracked along the way;

- how and when he/she will get further updates about progression.

First, fundamental step for MOC is focused in getting full support from right sponsorship, and namely from Sales Management. Changes requested are so fundamental across Sales Organization that only a full endorsement and support by top management can guarantee success of the overall project.

A second point to take in account is the involvement of all stakeholders. In order to identify all relevant ones, it is important to consider nature of current organizations, reflecting on potential consequences and impacts of Global Account Management Project on existing functions and businesses. Specific perspectives to be taken in account are (see Figure 2):

- lines of business;

- geographies;

- routes to market;

- selling motions.

Figure 2: Map of Potential Stakeholders to be Considered in an M.O.C Plan

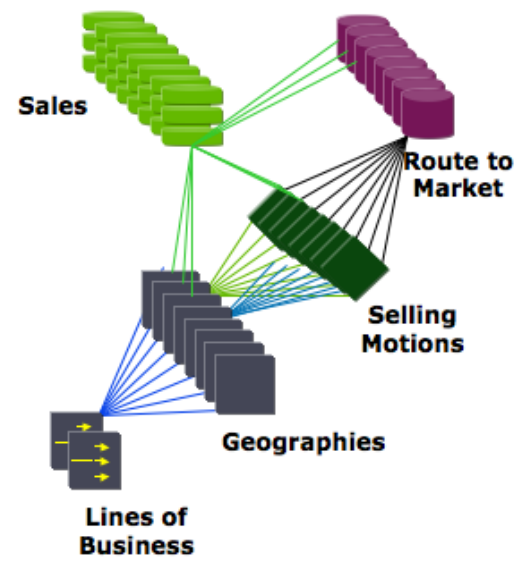

For each one of these components identification of possible roadblocks and potential sponsors it's of capital importance, along with accurate mapping of all 
stakeholders. Creating a chart of these points of influence, and evaluate risks and opportunities associated with each of them is strongly recommended.

Third step is to design and put in action governance which allows identifying clear ownership and responsibility for key decision making; frequency and format for Project Boards and Steering Committees; and flow and frequency of communication to keep constantly informed key decision makers.

Last, but absolutely fundamental component is the preparation and deployment of a comprehensive and consistent communication plans, which identifies:

- communication segmented target;

- communication frequency and format;

- speakers/senders owning each communication element;

- desired interactivity level in communication sessions.

Communication importance and (lack of) communication consequences are often under evaluated in large and complex transformation projects: so we feel the need of stressing once again the importance of devoting the right time and energy to the creation and the execution of a solid communication plan. Large transformations can be successful only if all the people involved in the changes are kept informed, constantly involved and have the opportunity to provide inputs and feedbacks. Significant part of this communication needs to come from Executive Sponsor, as part of his/her commitment to the success of the initiative. It is a major additional effort, but definitely it pays back in terms of speed of implementation and in preventing potential emerging obstacles. Communication is never enough, and has to be consistent over time and carefully planned upfront.

\subsection{Deployment}

In this chapter we will address deployment, both in terms of the first phase, so what we call "starting the journey", and from the perspective of leveraging progresses and constantly re-launch the overall actions, building on intermediate success.

\subsubsection{Starting the Journey}

Once the organization has finished preparing the foundation for the transformation, it's time to start. While it is key to have a thorough preparation, it's also important to move to execution mode quickly, to keep momentum and to leverage ideas and energies which have been created. Further thinking and adjustments which could postpone deployment should be avoided, as they can significantly reduce the trust created across all teams.

Two preliminary choices are of capital importance: put together a team of skilled, empowered and motivated individuals responsible to drive the implementation; and focus the first steps on finding and harvesting "low hanging fruits", i.e. quick actions which, by providing significant return, increase motivation and sense of achievement both for the driving team and for the whole organization.

After having assigned competent and well prepared individuals as Account Managers to selected Global Accounts, it's time to set associated targets 
(quantitative and clear; challenging but achievable) to each Account team. Account teams start putting together their Account Plans, and proactively working on Accounts to generate business opportunities. Extreme rigor need to be devoted by sales management in driving Opportunity Pipeline, and in managing it proactively with the goal of maximizing winning prospects. Executive sponsors should be assigned and made active to each one of the accounts. Comprehensive approach to customer feedbacks has to drive each action, with the goal of tuning and adjusting approach very quickly, while focusing on customer benefits and needs. And each one of this step needs to feed the other in iterative mode (see Figure 3 ).

Figure 3: An Iterative Model to Implement Key Initiatives to Drive Success

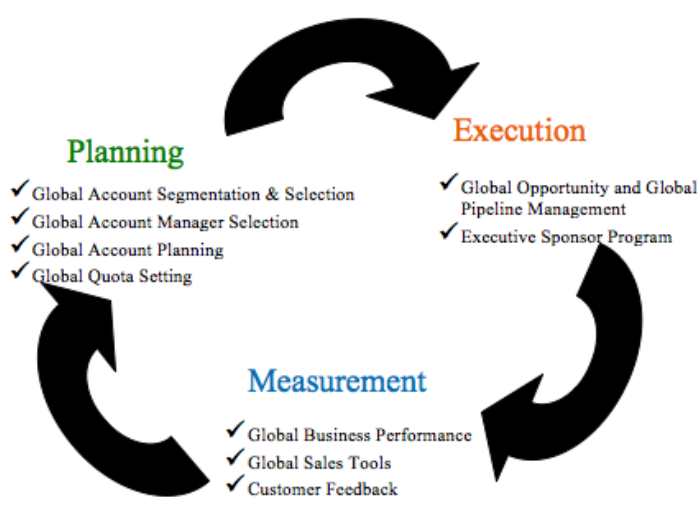

The whole new structure start working: sales tools simplifying sales reps life, while keeping major steps of sales process monitored and tracked, needs to be released and deployed. Constant support to front end organization and accurate monitoring of customer feedbacks need to be coupled to rigorous tracking of main business metrics and proactive action taking for fixing potential gaps created during implementation.

\subsubsection{Building from Success}

Complex transformations need to be constantly monitored in terms of progression. Quick and decisive corrective actions must be taken as soon as deviations start appearing on the dashboard. We actually suggest creating set of linked dashboards, similar in format but tailored to different levels of the organization, in order to make visible to everybody a coherent and timely view of the progresses and of consequent corrective actions.

The other area where we recommend focusing on is related to best practices which will be identified as transformation progresses. The ability to capture them and to replicate on broader base can dramatically speed up and make easier the whole implementation. Same comment for processes and tools: quickly detecting working areas and potential issues and fixing/improving them accordingly can significantly increase efficiency of hundreds of people working in front of the customers. 


\section{Conclusion}

Globalization of process, governance and decision making of large multinational companies is a solid, continuous trend in today business environment. Related changes can be leveraged as great opportunity for reinforcing or re-launching fruitful business relationship, and creating efficiency and advantages both for suppliers and customers. Suppliers who want to embrace those opportunities need to re-think their customer approach, namely in terms of set up of their sales force, and more specifically planning, a transformation from national based to international managed sales organization for those specific customers. Such an organizational transformation requires a careful and detailed preparation and excellence in execution. Ability to clearly design the end state, to size expected returns, to choose the right people to drive it, and to carefully execute and monitor are of capital importance for creating a successful new set-up.

During such a transition, risks exist and need to be carefully mitigated. On the other side we suggest to deeply reflect on the major risk which can jeopardize future success of an effective organization: maintaining organization in a status quo, while customers are evolving rapidly, across borders, creating a new environment which could become very difficult to be addressed through current set-up. 